\title{
An IPv6-Based Application Layer Protocol for Wireless Sensor Networks
}

\author{
Heng Wang \\ Key Laboratory of Industrial Internet of Things and \\ Networked Control \\ Chongqing University of Posts and \\ Telecommunications \\ Chongqing, China \\ wangheng@cqupt.edu.cn \\ Hang Yang \\ Key Laboratory of Industrial Internet of Things and \\ Networked Control \\ Chongqing University of Posts and \\ Telecommunications \\ Chongqing, China \\ 962558943@qq.com
}

\author{
Ping Wang \\ Key Laboratory of Industrial Internet of Things and \\ Networked Control \\ Chongqing University of Posts and \\ Telecommunications \\ Chongqing, China \\ wangping@cqupt.edu.cn
}

\begin{abstract}
IPv6 (Internet Protocol Version 6) is the next generation of IP protocol used to replace the current version of IP protocol designed by IETF(Internet Engineering Task Force),and applying IPv6 technology to the wireless sensor networks (WSN) will be a hot spot of research. Combining with the characteristics of IPv6 and wireless sensor networks, by analyzing the importance of data interaction methods for wireless sensor networks based on IPv6 in application layer, the application layer data interaction methods for IPv6 wireless sensor networks are presented in this paper. According to the actual needs of wireless sensor networks based on IPv6, the application layer data interaction methods are specific designed and implemented, which mainly include application layer communication mode and object relationship built. And the test results show that wireless sensor networks application layer data exchanges oriented to approach a stable communication between devices, improve the reliability of the communication process, and immediate issues.
\end{abstract}

Keywords-WSN; application layer; IPv6; communication mode;6LoWPAN

\section{INTRODUCTION}

IPv6 is designed by IETF (Internet Engineering Task Force) which is used to replace the current internet protocol version[1,2]. And it is the core of the next generation internet protocol, which has abundant address resources, strung expandability, high security and so on. So IPv6 technology applies to the wireless sensor network will be a hot spot of research[3,4]. Thus IETF sets up working groups which researches wireless sensor networks based on IPv6 technology and the development of standards. 6LoWPAN (IPv6 over Low power Wireless Personal Area Networks) protocol standard for wireless sensor network become a major application of IPv6 technology $[5,6]$. However, 6LoWPAN protocol standard did not define the functions and data interaction methods for application layer protocol.

Although the 6LoWPAN protocol stack defines the application layer protocol of CoAP (Constrained Application Protocol), which uses similar technology to HTTP (Hyper Text Transfer Protocol) protocol[7,8]. On the other hand, the CoAP protocol only applies to the resource-constrained devices, and it did not suitable for wireless sensor networks. In order to implement the communication among nodes in wireless sensor networks based on IPv6, data interaction methods should be designed in application layer so that the gateway and the nodes can communicate momentarily[9]. In this paper, we define three methods to solve the problem of data interaction in wireless sensor networks based on IPv6, including communication modes and object relationship built. Furthermore, we present the design and implementation of our methods as they are applied to wireless sensor networks based on IPv6.

\section{The TOPOLOGY OF Wireless SENSOR NETWORKS BASED ON IPV6}

The communication protocol stack of wireless sensor networks based on IPv6 supports a variety of network topologies, such as star topology, mesh topology and so on. It uses mesh topology which supports multi-hop communication and easily to be extended[10]. The topology of wireless sensor networks based on IPv6 is shown in Fig.1.

Devices in networks include nodes, routers, a gateway and the network manager. Nodes are primarily responsible for data collection, and send the data to the network manager through the gateway. On the other hand, the network manager can transmit command to nodes. Among 
the networks, the role of the gateway is to convert protocols.

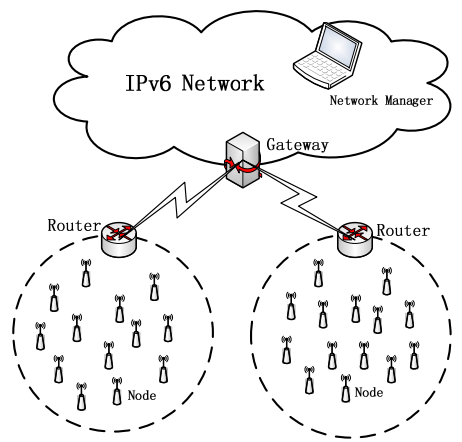

Figure 1. Topology of wireless sensor networks based on IPv6

\section{DATA INTERACTION METHODS}

The data interaction methods in wireless sensor networks based on IPv6 combine with wireless sensor networks, IPv6 technology and application layer protocol. The methods improve the reliability and stability of data transmission in industrial wireless networks. In this section, we introduce the data interaction methods including communication modes and the object relationship built.

\section{A. Communication Modes.}

Communication modes in application layer mainly provide data communication services for devices in networks to achieve the transfer of application entities data unit. In wireless sensor networks, communication modes mainly in the following three ways: Client/Server communication mode, Publisher/Subscriber communication mode, Report/Sink communication mode.

1) Client/Server communication mode. Client/Server communication mode is designed to allow the network manager to read, write or execute parameters to nodes. Client/Server communication mode supports the network manager by using three services: Read service that used to get attribute values of data object; Write service is a service that designed for data object, which means that data object can be written to the corresponding value to be their attribute values by write service; Execute service indicates that the network manager can perform an operation to nodes under the same networks. According to the application layer common frame format, the difference among the three kinds of service of the client/server communication mode is the payload frame format. In this paper, we introduced frame format of the read service as an example. When the network manager wants to adopt read service of attribute, it should send request of read service to nodes. The frame format of the request is shown in Fig.2.

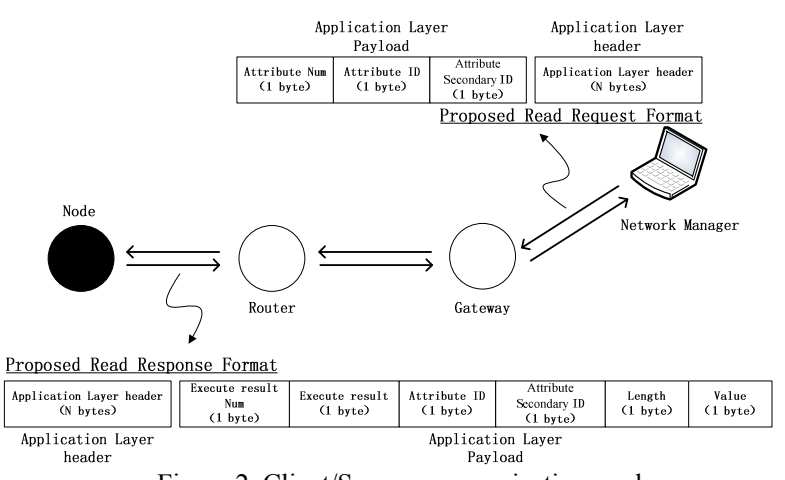

Figure 2. Client/Server communication mode

While nodes receive the request of read service, they analyze the request and send the response of read service to the network manager. The frame format of the response is shown in Fig.2. At this point, the read service of the client/server communication mode has been completed between the network manager and nodes.

2) Publisher/Subscriber communication mode. Publisher/Subscriber communication mode is mainly used to pre-configure nodes periodicity report the data they collected to the network manager. The frame format of the publisher/subscriber communication mode in application layer is shown in Fig.3.

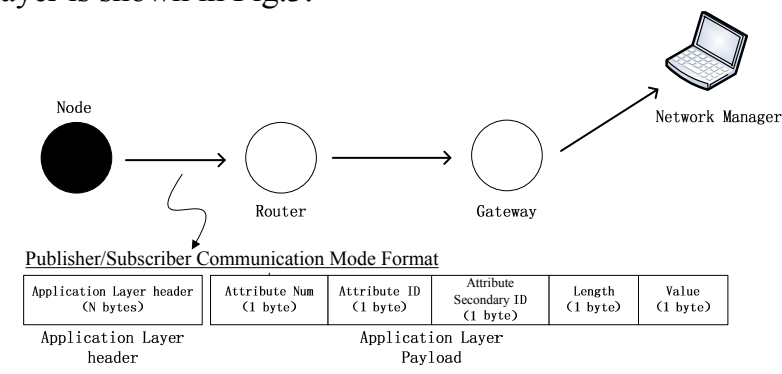

Figure 3. Publisher/Subscriber communication mode

3) Report/Sink communication mode. When nodes detect an error occurs in networks, they use report/sink communication mode to send the error information to the network manager. The frame format of the report/sink communication mode in application layer is shown in Fig.4.

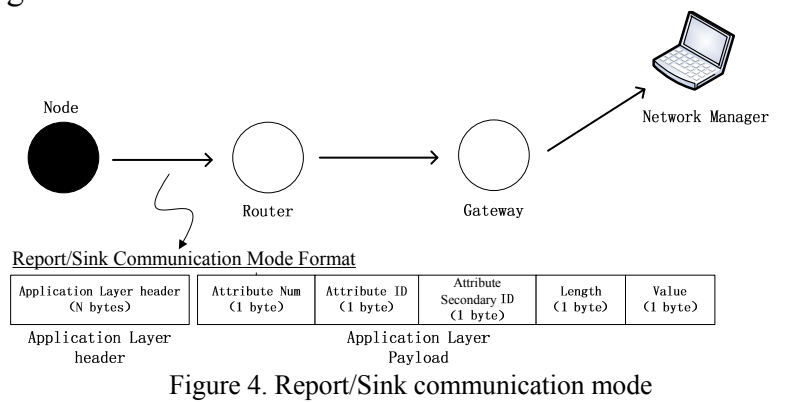

B. Object Relationship Built.

March 2010, the CORE working group of IETF began to develop the CoAP protocol. The CoAP protocol is an application layer which used for resource-constrained devices. The core content of the CoAP protocol is resource discovery, which is mainly used for self-discovery and discovery a list of resources on devices. However, the function of resource discovery is not suitable for wireless sensor networks. In addition, the relevant standard for 
wireless sensor networks has developed similar function to resource discovery. Meanwhile, some devices want to exchange data through the network so that to control other devices. In order to increasing the interoperability of the entire network, we design object relationship built of wireless sensor networks based on IPv6 to achieve data interaction between any devices in networks. To achieve object relationship built, there have one way which is online configuration.

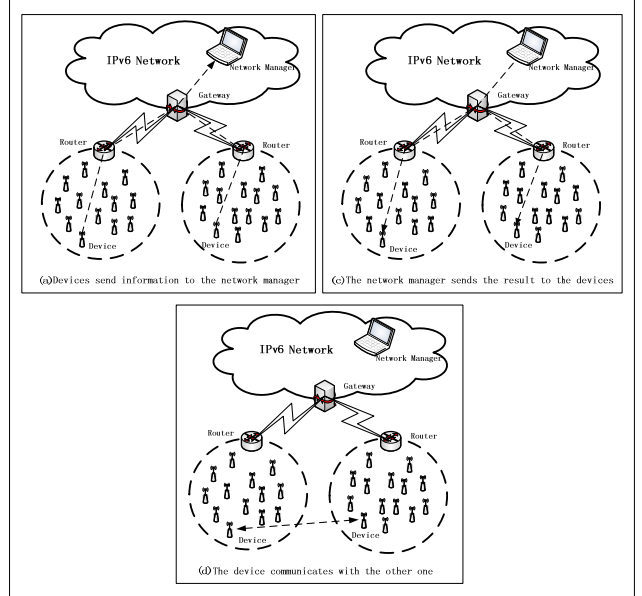

Figure 7. The process of online configuration

Online configuration depends on the network manager to establish the object relationship between any devices in networks. The process of online configuration is shown in Fig.7. When the device joins networks, it sends a frame contains the device information to the network manager. And the network manager keeps the device information it receives in table. After receiving all the device information from the devices, the network manager begins to establish the object relationship and sends the result to the devices. Then one device which receives the result can communicate with the other one.

\section{EXPERIMENT AND EVALUATION}

In this paper, we perform a small-scale test on the data interaction methods in wireless sensor networks based on IPv6. The test nodes include a gateway, two routers and six nodes. According to the topology of wireless sensor networks based on IPv6, we set up a test system as shown in Fig.9. And the topology of the system is shown on the interface of the network manager as Fig.10. We use the network manager and the packet sniffer software to analyze the data which devices send or receive. Thus we can test the feasibility of the methods by comparing the data.

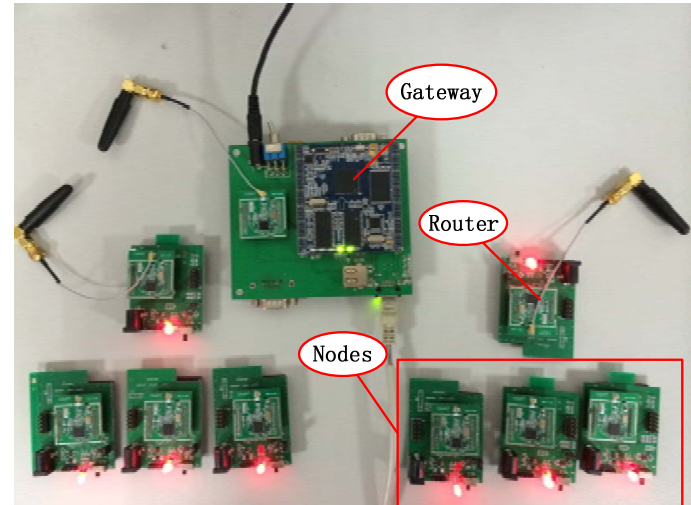

Figure 9. The test system

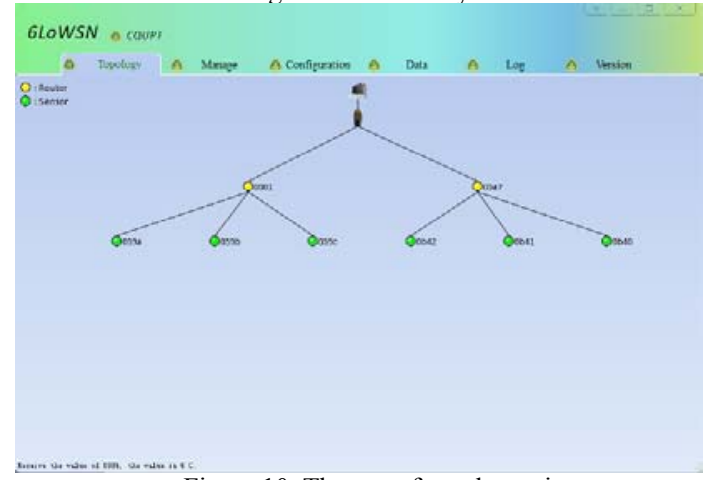

Figure 10. The test of topology view

\section{A. The Test of Communication Modes}

Define abbreviations and acronyms the first time they are used in the text, even after they have been defined in the abstract. Abbreviations such as IEEE, SI, MKS, CGS, $\mathrm{sc}$, dc, and rms do not have to be defined. Do not use abbreviations in the title or heads unless they are unavoidable.

The test of communication modes is going to compare the data which the network manager receives and the device sends. So we need to test in two aspects:(1)The network manages sends the request to the device;(2) The device sends the response to the network manager.
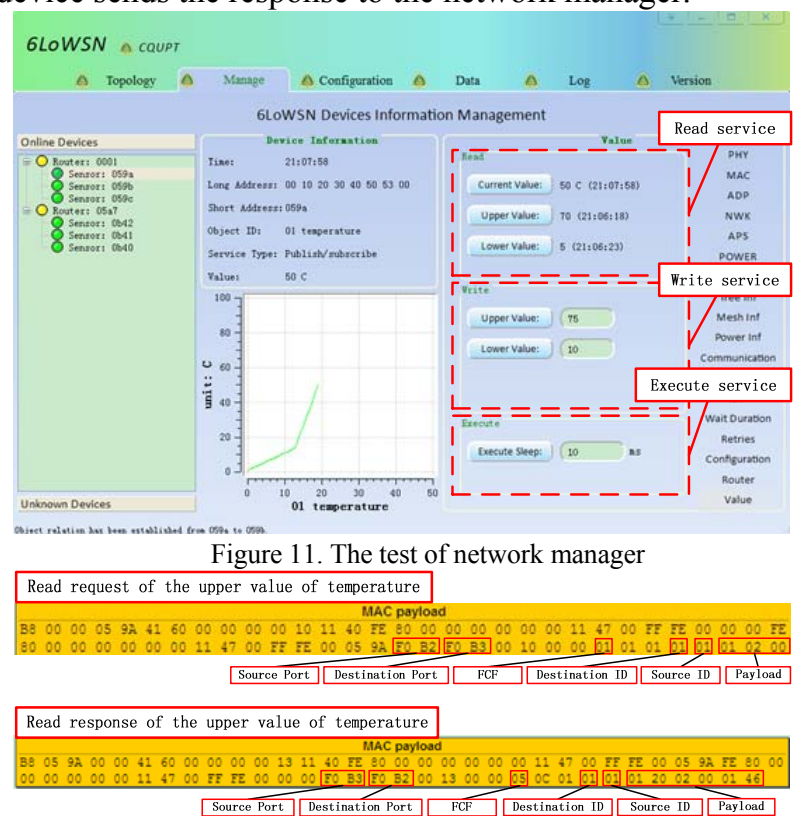

Figure 12 . The analysis of read request and response 
The data is shown on the interface of the network manager as shown in the Fig .11. The command information of request and response is shown in Fig. 12. From the graphs we can see that the upper value of temperature which the network manager receives and the device sends is the same.

\section{B. The Test of Object Relationship Built}

Object relationship built can ensure the communication among devices freely in networks. Whether the object relationship built can be used in wireless sensor networks based on IPv6, there have two aspects need to be tested:(1) The sender need to get the information of the receiver;(2) The sender can communicate with the receiver.

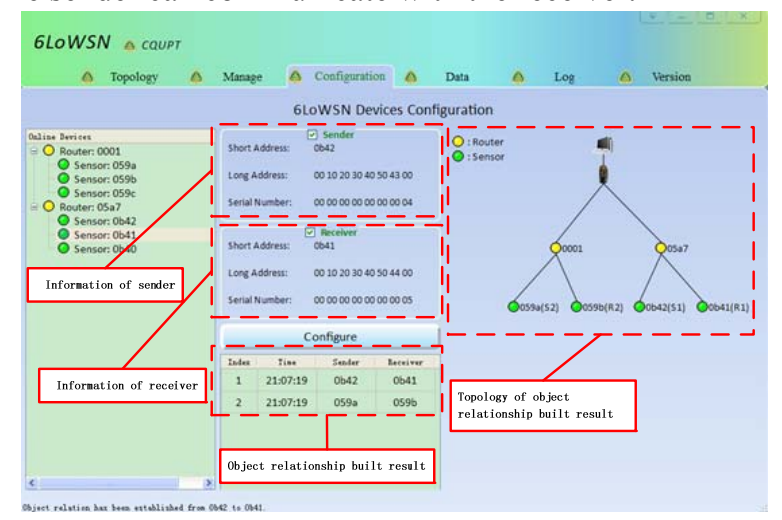

Figure 15. The result of object relationship built

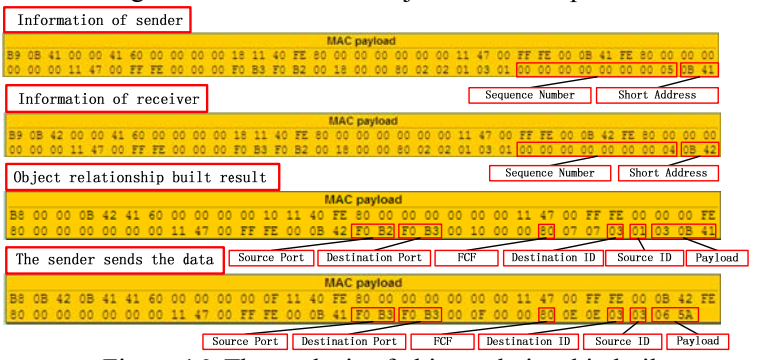

Figure 16. The analysis of object relationship built

As shown in Fig.15 and Fig.16, the sender gets the information of receiver. The receiver receives the data which the sender sends. And the result of object relationship built is shown on the interface of the network manager as show in Fig.15.

\section{CONCLUSIONS}

In this paper, we present the data interaction methods in wireless sensor networks based on IPv6. The data interaction methods contain communication modes and the object relationship built. Communication modes can be used to the different application environment. Object relationship built achieves that devices can communicate with anyone in networks. As our test results shown, the data interaction methods can meet the needs of wireless sensor networks based on IPv6.Authors and Affiliations

\section{ACKNOWLEDGMENT}

This work was supported by the National High Technology Research and Development Program of China (Grant No. 2015AA043801), and the Fundamental and Advanced Research Program of Chongqing (Grant No. cstc2013jcyjA40008).

\section{REFERENCES}

[1] WANG XIAONAN, GAO DEMIN. An IPv6 Address Configuration Scheme for All-IP Wireless Sensor Networks[J]. Ad-hoc \&amp; sensor wireless networks, 2011, 12(3/4): 209-227.

[2] Stanford L. Levin, Stephen Schmidt. IPv4 to IPv6: Challenges, solutions, and lessons[J].Telecommunications Policy,2014,12:10591068 .

[3] S. Deering, R. Hinden. Internet Protocol, Version 6 (IPv6) Specification[S]. RFC 2460. December 1998

[4] Lu ZHAO, Guang-wei BAI, Hang SHEN, Zhen-min et al.Prioritybased IEEE 802.15.4 CSMA/CA mechanism for WSNs[J]. The Journal of China Universities of Posts and Telecommunications.2013,2:47-53.

[5] SANARAS I K,HASSAPIS G D,GIALELIS J V, et al.A Modified DPWS Protocol Stack for 6LoWPAN-Based Wireless Sensor Networks[J]. IEEE transactions on industrial informatics, 2013, 9(1): 209-217

[6] Xiaonan Wang, Deguang Le, Yufeng Yao, et al.Location-based mobility support for 6LoWPAN wireless sensor networks[J]. Journal of Network and Computer Applications.2015,4:68-77.

[7] Angelo P. Castellani, Michele Rossi, Michele Zorzi.Enabling endto-end CoAP-based communications for the Web of Things. Ad Hoc Networks.2014,7:71-84.

[8] Angelo P. Castellani, Michele Rossi, Michele Zorzi. Back pressure congestion control for CoAP/6LoWPAN networks[[J]. Ad Hoc Networks.2014,11:71-84.

[9] YANG SOOYOUNG, PARK SUNGJIN, LEE EUU JU, et al. Dual Addressing Scheme in IPv6 over IEEE 802.15.4 Wireless Sensor Networks[J].ETRI journal, 2008, 30(5): 674-684.

[10] HU TING, CUI XIAOYAN, YAO YAN. A new interconnection scheme for WSN and IPv6-based internet[C]. Information, Computing and Telecommunication, 2009. YC-ICT '09. IEEE Youth Conference on 2009: 34 - 37. 